\title{
The Use of Epidemiological Studies for Health Risk Assessment
}

\author{
Kevin Fong-Rey Liu, Wei-Ru Chen, Yu-Chen Yeh, Li-Wen Chang, and Yung-Shuen Shen
}

\begin{abstract}
The hazard quotient (HQ), the ratio of the intake of the substance to its reference dose, is usually used in the health risk assessment (HRA) of a noncarcer substance. However, the values of HQ cannot inform any specific human health effect. Compared to HQ, using epidemiological studies in HRA can improve the defect. This study attempts to examine the potential of the use of epidemiological studies for HRA. Previous epidemiological studies help to construct the linear concentration-response relationships between air pollutants and associated diseases and two case studies are used to demonstrate this method.
\end{abstract}

Index Terms-Epidemiological studies, health risk assessment, air pollution, sandstorm.

\section{INTRODUCTION}

A human health risk assessment (HRA) is the process to estimate the nature and probability of adverse health effects in humans who may be exposed to chemicals in contaminated environmental media, now or in the future. Human health risk assessment includes 4 basic steps (Fig. 1): hazard identification, dose-response assessment, exposure assessment and risk characterization.

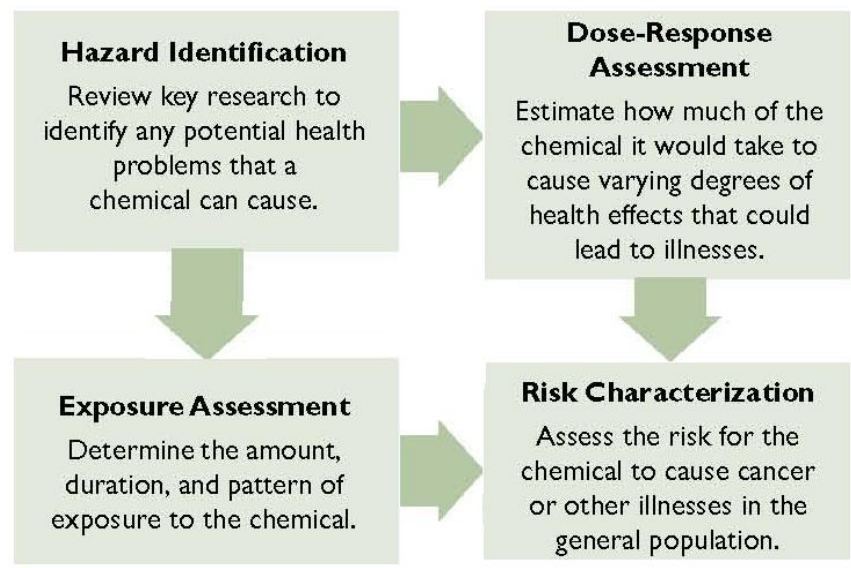

Fig. 1. The four basic steps in health risk assessment [1].

In the first step, hazard identification, scientists determine the types of health problems a chemical could cause by reviewing studies of its effects in humans and laboratory animals. In dose-response assessment, scientists evaluate the information obtained during the hazard identification step to

Manuscript received January 13, 2013; revised March 13, 2013.

Kevin Fong-Rey Liu, Wei-Ru Chen, Yu-Chen Yeh, Li-Wen Chang are with the Department of Safety, Health and Environmental Engineering, Ming Chi University of Technology, New Taipei City, Taiwan (e-mail: kevinliu@mail.mcut.edu.tw; U98167018@mail2.mcut.edu.tw; U98167026@mail2.mcut.edu.tw; U98167033@mail2.mcut.edu.tw).

Y. S. Shen is with the Holistic Education Center, Mackay Medical College, New Taipei City, Taiwan (e-mail: ysshen@mmc. edu.tw). estimate the amount of a chemical that is likely to result in a particular health effect in humans. In exposure assessment, scientists attempt to determine how long people were exposed to a chemical; how much of the chemical they were exposed to; whether the exposure was continuous or intermittent; and how people were exposed - through eating, drinking water and other liquids, breathing, or skin contact. The last step in risk assessment brings together the information developed in the previous three steps to estimate the risk of health effects in an exposed population. In the risk characterization step, scientists analyze the information developed during the exposure and dose-response assessments to describe the resulting health risks that are expected to occur in the exposed population. This information is presented in different ways for cancer and non-cancer health effects.

Although the second step refers to the "dose-response" relationship, the same principles generally apply for studies where the exposure is to a concentration of the agent (e.g., airborne concentrations applied in inhalation exposure studies), and the resulting information is referred to as the "concentration-response" relationship. Usually, the relationship is based on observed data from human clinical trials, epidemiologic studies, animal tests and cell studies, which order also represents the order of their precisions in the dose-response relationship, from better to worse.

Nachman et al. [2] and Raffaele et al. [3] have discussed the use of epidemiologic data in HRA and highlighted some of the advantages and limitations. Nachman et al. [2] learned the lessons from some case studies and suggested the use of epidemiologic data to determine noncancer quantitative dose-response relationships. Raffaele et al. [3] also learned the lessons from their case studies and identified a number of important benefits to be gained by using epidemiology data in HRA: 1) identification of specific human health effects that were not identified in animal models (e.g., inorganic arsenic); 2) affirmation of the human relevance of effects identified in animal models (e.g., neurodevelopmental effects seen with chlorpyrifos); 3) ability to evaluate health effects for which animal models are unavailable or limited (e.g., inorganic arsenic and cancer, pesticides and parkinson's disease); 4) evaluation of health effects in the range of expected human exposures (e.g., inorganic arsenic); 5) utility of biomonitoring information (chlorpyrifos). They also identified a number of important barriers: 1) difficulty in converting human biomonitoring information to exposure levels that can be confidently associated with a specific hazard (difficulties related both to back-calculating from measured tissue levels to exposure levels as well as to understanding the source of measured metabolites [i.e. direct 
exposure to metabolite versus products of internal metabolism], chlorpyrifos); 2) difficulty in identifying exposed populations (both with respect to identifying the specific compounds to which exposure occurred and which individuals were exposed; parkinson' disease and pesticides); 3) difficulty in isolating specific exposures (both routes of exposure and quantification of exposure; all three examples); 4) concerns regarding appropriateness of evaluated population (in the key arsenic studies, including diet, genetics, alternative exposure sources and the issue of the younger age of the control group).

This study attempts to examine the potential of the use of epidemiological studies for HRA. According to some epidemiological studies [4], [5], the linear concentration -response relationships between air pollutants and associated diseases are constructed and they are thereby applied to two case studies. The first case identifies the morbidities of the related diseases of the five metropolises (Taipei, New Taipei, Taichung, Tainan and Kaohsiung) in Taiwan during 2011, as shown in Fig. 2; the second case identifies the morbidities of the related diseases during a sandstorm of 2010 from China.

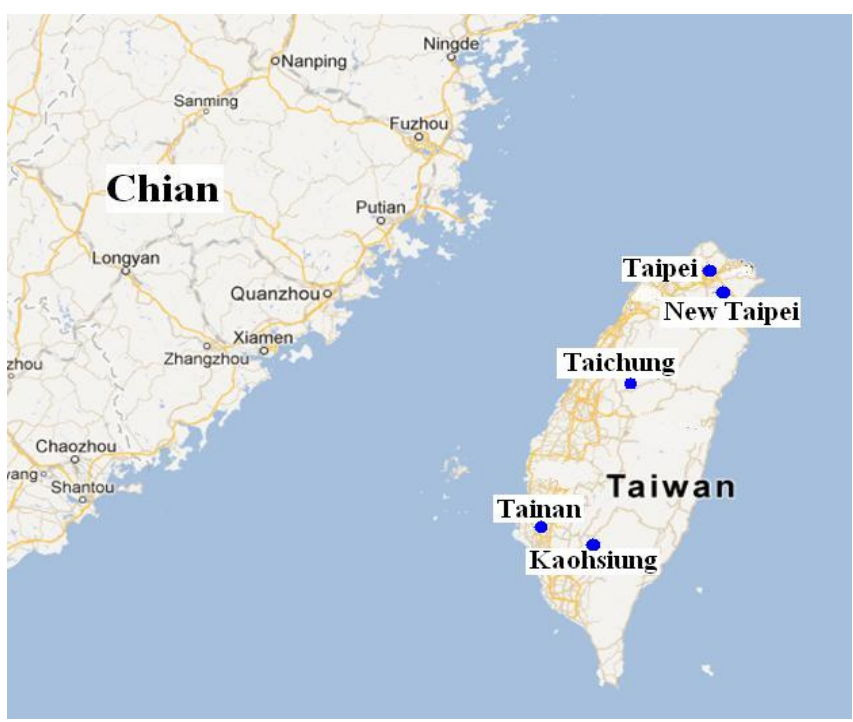

Fig. 2. Taiwan map.

\section{Methods AND MATERIALS}

\section{A. Hazard Identification}

The major air pollutants are total suspended particulates (TSP), sulfur dioxide $\left(\mathrm{SO}_{2}\right)$, nitrogen dioxide $\left(\mathrm{NO}_{2}\right)$ and carbon monoxide (CO). According to Liu and his colleagues' work (2012), the health effects of the four air pollutants are shown in Fig. 3. TSP can lead to cardiovascular diseases, bronchitis, lung cancer, pneumonia, emphysema, chronic obstructive pulmonary disease (COPD), chronic airway obstruction (CAO) and asthma; $\mathrm{SO}_{2}$ induces bronchitis, lung cancer, pneumonia, emphysema, $\mathrm{COPD}$ and $\mathrm{CAO} ; \mathrm{NO}_{2}$ can cause cardiovascular diseases, bronchitis, lung cancer, pneumonia, emphysema and COPD; and exposure to CO can result in cardiovascular diseases and CAO. Bronchitis is inflammation of the mucous membranes of the bronchi, the airways that carry airflow from the trachea into the lungs. Asthma is a common chronic inflammatory disease of the airways characterized by variable and recurring symptoms, reversible airflow obstruction, and bronchospasm. Lung cancer is a disease of uncontrolled cell growth in tissues of the lung and may lead to metastasis to adjacent tissues. Pneumonia is an inflammatory condition of the lung, including lung parenchyma and abnormal alveolar filling with fluid. Emphysema is a long term, progressive disease of the lung that primarily causes shortness of breath. CAO is a type of pulmonary disorder. COPD refers to a group of diseases that cause airflow blockage and breathing-related problems, including chronic bronchitis, emphysema and chronic obstructive airways diseases. Acute bronchitis typically follows upper respiratory tract infections such as influenza and often leads to pneumonia. It is not recognized that chronic bronchitis can turn into lung cancer or emphysema; however it may weaken people so that they can easily get lung cancer or emphysema. Usually, bronchitis and asthma will cause $\mathrm{CAO}$ and they together with emphysema can result in COPD. The above respiratory diseases are related to some extent and their cause-effect relationships can be established in Fig. 3.

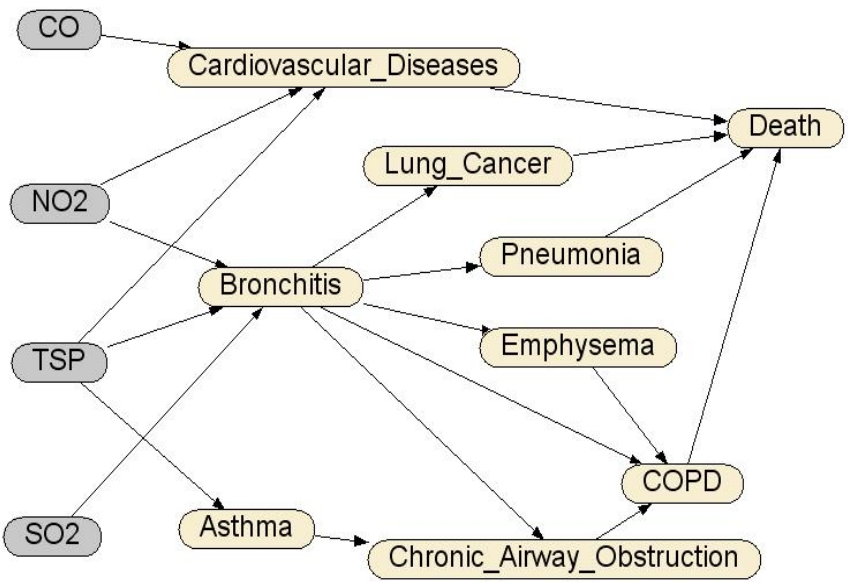

Fig. 3. HRA structure for air pollution [4]

\section{B. Dose Response Assessment}

The relationships between human morbidity or mortality and the four air pollutants have been discussed in Liu and his colleagues' work [4]. Each increase in $100 \mu \mathrm{g} / \mathrm{m}^{3}$ of TSP results in a $6.58 \%$ increase in mortality; an incremental difference of $100 \mu \mathrm{g} / \mathrm{m}^{3}$ (0.049 ppm) of $\mathrm{NO}_{2}$ is associated with a $3.95 \%$ increase in mortality and a $63.63 \%$ increase in bronchitis morbidity; an elevation of $\mathrm{SO}_{2}$ of $100 \mu \mathrm{g} / \mathrm{m}^{3}$ $(0.035 \mathrm{ppm})$ results in additional mortality and bronchitis morbidity by 8.2 and $63.63 \%$, respectively. Each $10 \mu \mathrm{g} / \mathrm{m}^{3}$ increment of $\mathrm{PM}_{10}\left(17.8 \mu \mathrm{g} / \mathrm{m}^{3}\right.$ of TSP) leads to a $1.4 \%$ increase in morbidities of cardiovascular diseases and a $3.4 \%$ increase in bronchitis and asthma. Every additional $1 \mathrm{ppm}$ of CO will increase mortality by $3.57 \%$ and cardiovascular diseases by $4.6 \%$; and each incremental difference of 0.010 ppm of $\mathrm{NO}_{2}$ will result in a $1.83 \%$ increase in morbidity of cardiovascular diseases. In Taiwan, the 2005 annual mean concentrations of TSP, $\mathrm{SO}_{2}, \mathrm{NO} 2$ and $\mathrm{CO}$ are $92.97 \mu \mathrm{g} / \mathrm{m}^{3}$, $0.005,0.018$ and $0.54 \mathrm{ppm}$, respectively. In that year, the morbidities of pneumonia, cardiovascular diseases, lung cancer, asthma, emphysema, COPD, CAO, bronchitis and air quality-related mortality are 3.01, 7.28, 0.13, 3.65, 0.11, 3.39, $0.96,4.12$ and $0.138 \%$, respectively. Accordingly, the 
functions of morbidity and mortality rates can be derived for each air pollutant, as shown in the last column of Table I.

\begin{tabular}{|c|c|c|}
\hline $\begin{array}{l}\text { Air } \\
\text { pollutant }\end{array}$ & Induced disease & $\begin{array}{l}\text { Functions of mortality or morbidity } \\
\text { rates }\end{array}$ \\
\hline \multirow{4}{*}{ TSP } & Mortality & $0.1338 \%\left[1+\frac{6.58 \%}{100}(\mathrm{TSP}-92.97)\right]$ \\
\hline & Bronchitis & $4.12 \%\left[1+\frac{3.4 \%}{17.8}(\mathrm{TSP}-92.97)\right]$ \\
\hline & $\begin{array}{l}\text { Cardiovascular } \\
\text { Diseases }\end{array}$ & $7.28\left[1+\frac{1.4 \%}{17.8}(\mathrm{TSP}-92.978)\right]$ \\
\hline & Asthma & $3.65 \%\left[1+\frac{3.4 \%}{17.8}(\mathrm{TSP}-92.97)\right]$ \\
\hline \multirow{2}{*}{$\mathrm{SO}_{2}$} & Mortality & $0.0812 \%\left[1+\frac{8.2 \%}{0.035}\left(\mathrm{SO}_{2}-0.005\right)\right]$ \\
\hline & Bronchitis & $4.12 \%\left[1+\frac{29.94 \%}{0.035}\left(\mathrm{SO}_{2}-0.005\right)\right]$ \\
\hline \multirow{3}{*}{$\mathrm{NO}_{2}$} & Mortality & $0.1338 \%\left[1+\frac{3.95 \%}{0.049}\left(\mathrm{NO}_{2}-0.018\right)\right]$ \\
\hline & Bronchitis & $4.12 \%\left[1+\frac{63.63 \%}{0.049}\left(\mathrm{NO}_{2}-0.018\right)\right]$ \\
\hline & $\begin{array}{l}\text { Cardiovascular } \\
\text { Diseases }\end{array}$ & $7.28\left[1+\frac{1.83 \%}{0.010}\left(\mathrm{NO}_{2}-0.018\right)\right]$ \\
\hline \multirow{2}{*}{$\mathrm{CO}$} & Mortality & $0.0571 \%\left[1+\frac{3.57 \%}{1}(\mathrm{CO}-0.54)\right]$ \\
\hline & $\begin{array}{l}\text { Cardiovascular } \\
\text { Diseases }\end{array}$ & $7.28 \%\left[1+\frac{4.6 \%}{1}(\mathrm{CO}-0.54)\right]$ \\
\hline
\end{tabular}

The morbidity rates in Table I are the single relationships between a pollutant and a disease. But it is very difficult to find the relationships between multiple pollutants and a disease. To obtain the overall morbidity of a disease, a technology of the fusion of probability has been proposed in [4]. For example, a disease caused by three pollutants $E_{1}, E_{2}$ and $\mathrm{E}_{3}$ is displayed as;

$$
\begin{aligned}
& P\left(H \mid E_{1} \cap E_{2} \cap E_{3}\right)=\frac{P\left(E_{1} \cap E_{2} \cap E_{3} \cap H\right)}{P\left(E_{1} \cap E_{2} \cap E_{3}\right)} \\
= & \frac{P\left(E_{1} \cap E_{2} \cap E_{3} \cap H\right)}{P\left(E_{1} \cap E_{2} \cap E_{3} \cap H\right)+P\left(E_{1} \cap E_{2} \cap E_{3} \cap \neg H\right)}
\end{aligned}
$$

where $P\left(E_{1} \cap E_{2} \cap E_{3} \cap H\right)=P\left(E_{1} \cap E_{2} \cap E_{3} \mid H\right) P(H)$ and assume that $\mathrm{e}_{1} 、 \mathrm{e}_{2} 、 \mathrm{e}_{3}$ are conditional independent, then

$$
\begin{aligned}
P\left(E_{1} \cap E_{2} \cap E_{3} \cap H\right) & =P\left(E_{1} \cap E_{2} \cap E_{3} \mid H\right) P(H) \\
& =P\left(E_{1} \mid H\right) P\left(E_{2} \mid H\right) P\left(E_{3} \mid H\right) P(H) \\
& =\frac{P\left(H \mid E_{1}\right) P\left(H \mid E_{2}\right) P\left(H \mid E_{3}\right) P\left(E_{1}\right) P\left(E_{2}\right) P\left(E_{3}\right)}{P(H)^{2}} \\
& =\frac{\prod_{i=1}^{3} P\left(H \mid E_{i}\right) \cdot \prod_{i=1}^{3} P\left(E_{i}\right)}{P(H)^{2}}
\end{aligned}
$$

Similarly,

$$
\begin{aligned}
& P\left(E_{1} \cap E_{2} \cap E_{3} \cap \neg H\right) \\
& =\frac{P\left(\neg H \mid E_{1}\right) P\left(\neg H \mid E_{2}\right) P\left(\neg H \mid E_{3}\right) P\left(E_{1}\right) P\left(E_{2}\right) P\left(E_{3}\right)}{P(\neg H)^{2}} \\
& =\frac{\left[1-P\left(H \mid E_{1}\right)\right]\left[1-P\left(H \mid E_{2}\right)\right]\left[1-P\left(H \mid E_{3}\right)\right] P\left(E_{1}\right) P\left(E_{2}\right) P\left(E_{3}\right)}{[1-P(H)]^{2}} \\
& =\frac{\prod_{i=1}^{3}\left[1-P\left(H \mid E_{i}\right)\right] \cdot \prod_{i=1}^{3} P\left(E_{i}\right)}{[1-P(H)]^{2}}
\end{aligned}
$$

$$
\begin{aligned}
& P\left(H \mid E_{1} \cap E_{2} \cap E_{3}\right)=\frac{\frac{\prod_{i=1}^{3} P\left(H \mid E_{i}\right) \cdot \prod_{i=1}^{3} P\left(E_{i}\right)}{P(H)^{2}}}{\frac{\prod_{i=1}^{3} P\left(H \mid E_{i}\right) \cdot \prod_{i=1}^{3} P\left(E_{i}\right)}{P(H)^{2}}+\frac{\prod_{i=1}^{3}\left[1-P\left(H \mid E_{i}\right)\right] \cdot \prod_{i=1}^{3} P\left(E_{i}\right)}{[1-P(H)]^{2}}} \\
& =\frac{\frac{\prod_{i=1}^{3} P\left(H \mid E_{i}\right)}{P(H)^{2}}}{\frac{\prod_{i=1}^{3} P\left(H \mid E_{i}\right)}{P(H)^{2}}+\frac{\prod_{i=1}^{3}\left[1-P\left(H \mid E_{i}\right)\right]}{[1-P(H)]^{2}}}
\end{aligned}
$$

when $\mathrm{i}=\mathrm{n}$

$$
P\left(H \mid E_{1} \cap \cdots \cap E_{n}\right)=\frac{\frac{\prod_{i=1}^{n} P\left(H \mid E_{i}\right)}{P(H)^{n-1}}}{\frac{\prod_{i=1}^{n} P\left(H \mid E_{i}\right)}{P(H)^{n-1}}+\frac{\prod_{i=1}^{n}\left[1-P\left(H \mid E_{i}\right)\right]}{[1-P(H)]^{n-1}}}
$$

\section{RESUlTS: RISK CHARACTERIZATION}

\section{A. Case 1}

There are five metropolises in Taiwan: Taipei, New Taipei, Taichung, Tainan and Kaohsiung. Although the HQs of the air pollution in the five cities are below one, which means it is unlikely to cause adverse health effects, but it is still an interesting topic to understand the health effects in the five cities, due to the air pollution. According to the data of 2011 from the air quality measurement stations, the first case study identifies the morbidities of the related diseases of the five metropolises during 2011, and the results are shown from Fig. 4 to 11 .

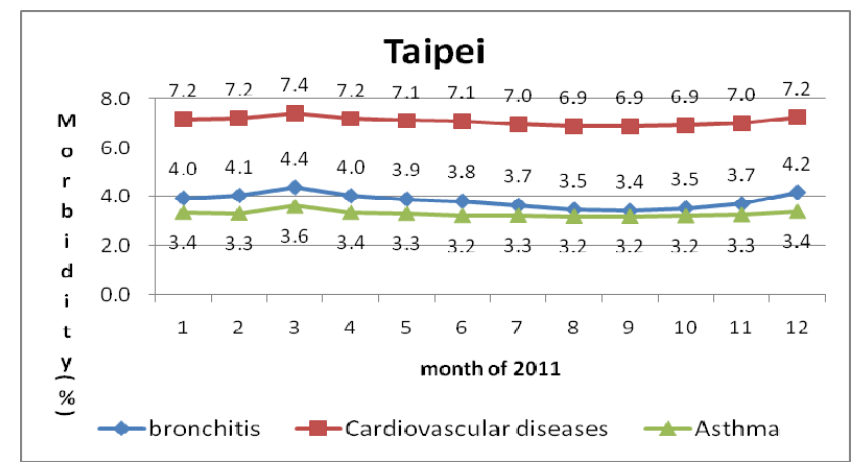

Fig. 4. The morbidities of the three diseases in Taipei, in 2011.

\section{B. Case 2}

In 2010, there is severe sandstorm from China, lasting from March 21 to March 23. According to the data from the Taishang air quality measurement station (in New Taipei), the second case study identifies the morbidities of the related diseases during the sandstorm. The results are shown from Fig. 12 to 17. 


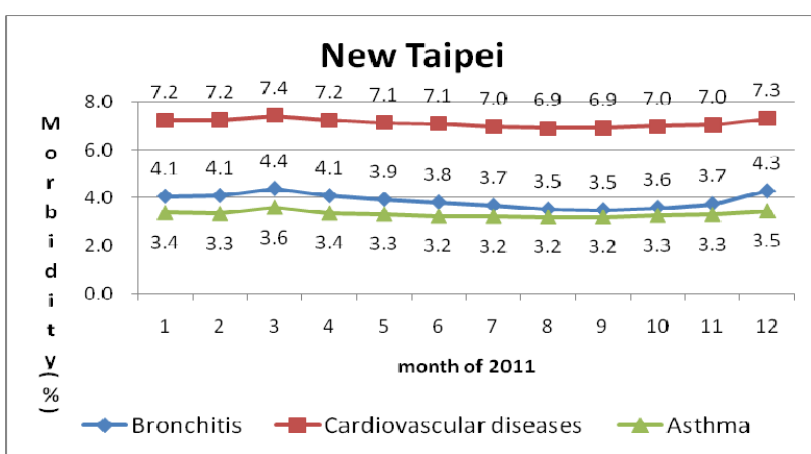

Fig. 5. The morbidities of the three diseases in New Taipei, in 2011.

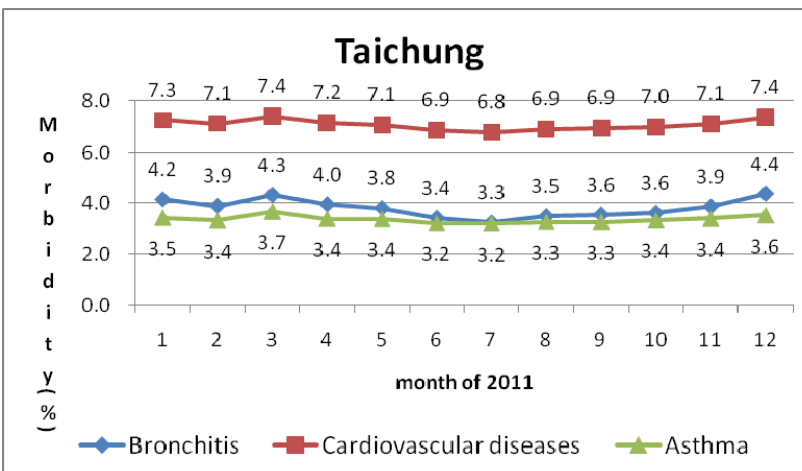

Fig. 6. The morbidities of the three diseases in Taichung, in 2011.

\section{Tainan}

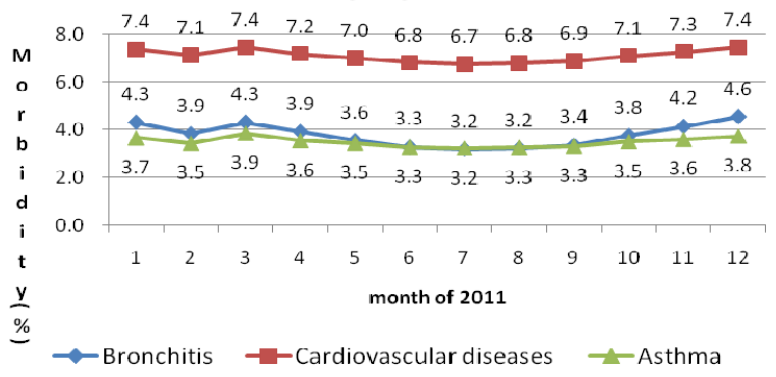

Fig. 7. The morbidities of the three diseases in Tainan, in 2011.

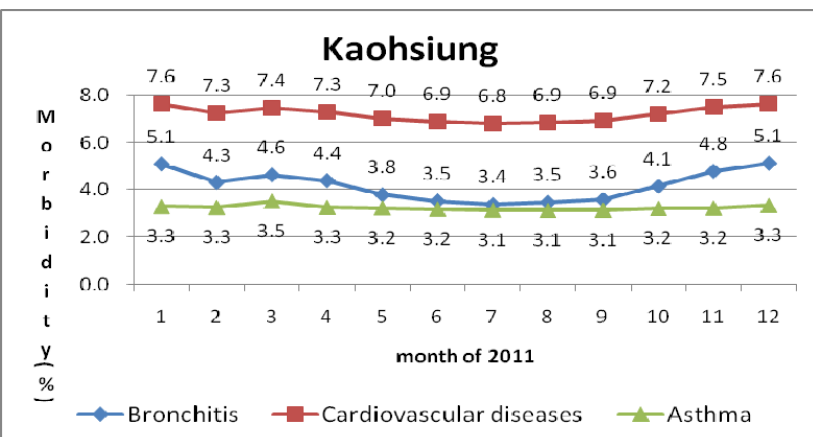

Fig. 8. The morbidities of the three diseases in Kaohsiung, in 2011.

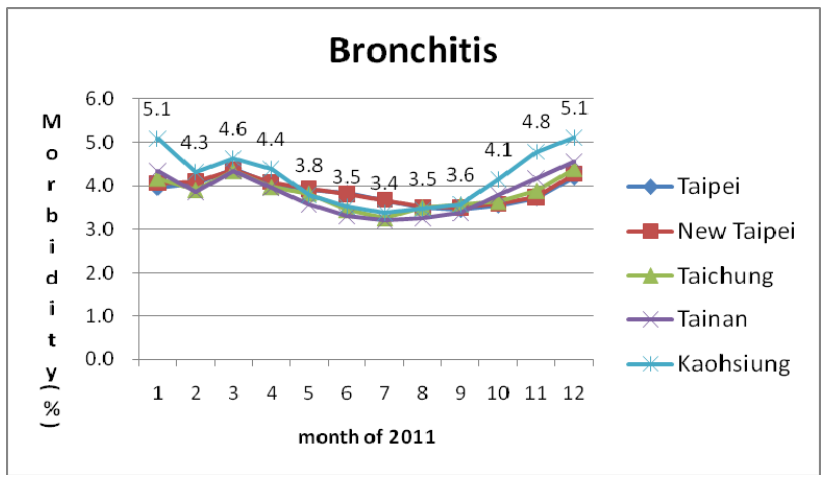

Fig. 9. The morbidities of bronchitis the five cities, in 2011.

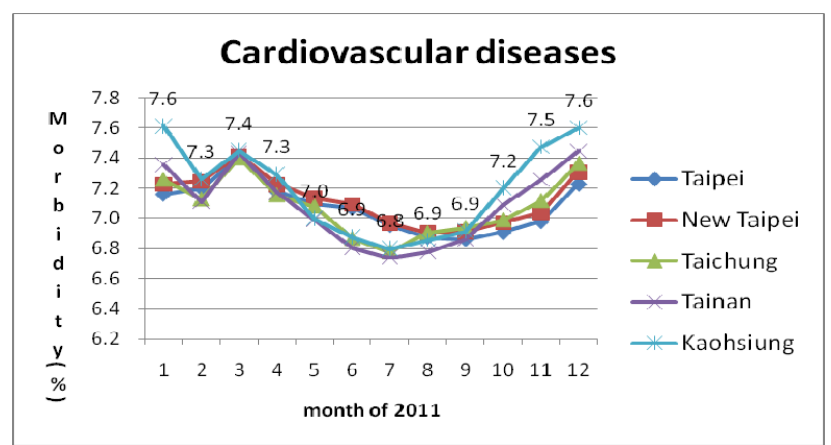

Fig. 10. The morbidities of cardiovascular diseases the five cities, in 2011.

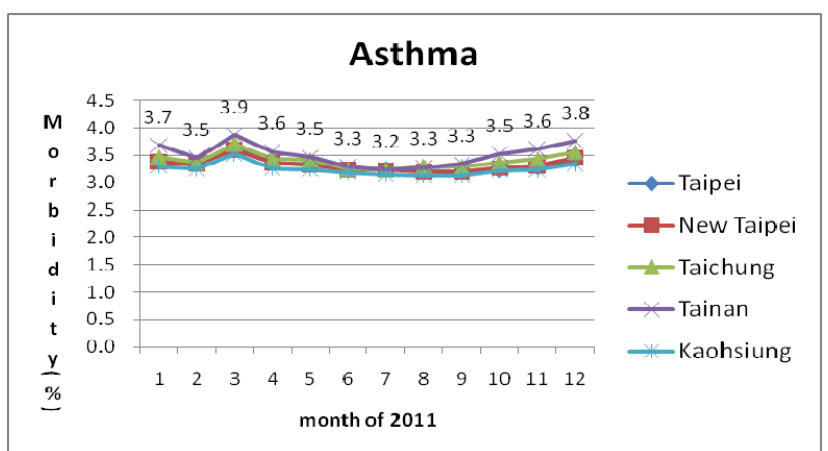

Fig. 11. The morbidities of asthma the five cities, in 2011..

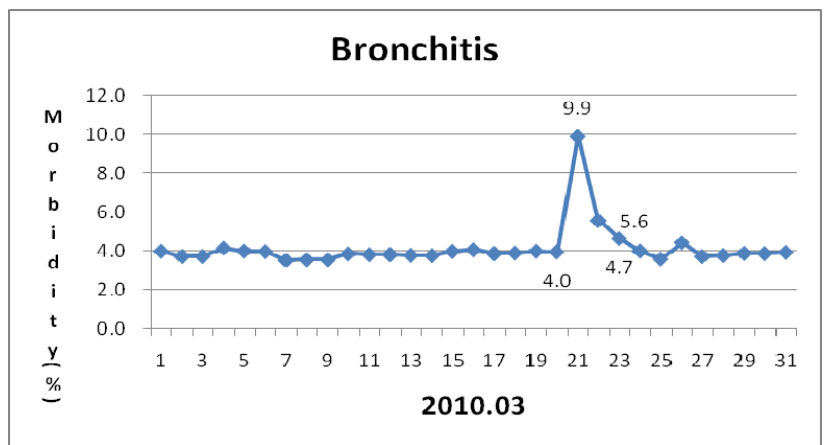

Fig. 12. The morbidities of bronchitis caused by $\mathrm{PM}_{10}$ during the sandstorm (2010.03.21 23).

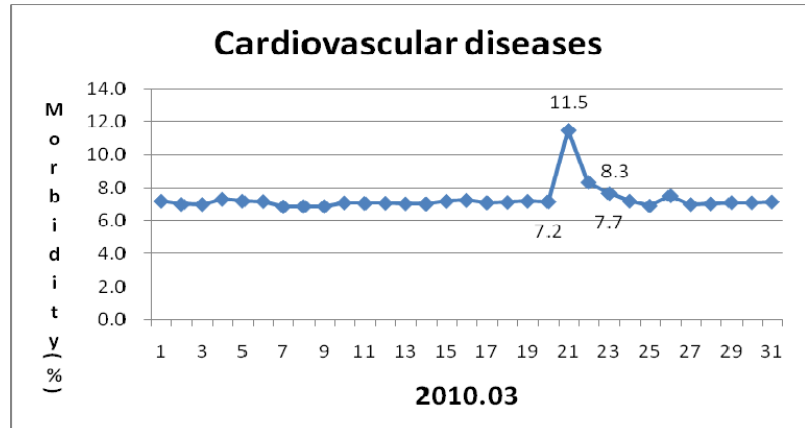

Fig. 13. T The morbidities of cardiovascular diseases caused by $\mathrm{PM}_{10}$ during the sandstorm (2010.03.21 23).

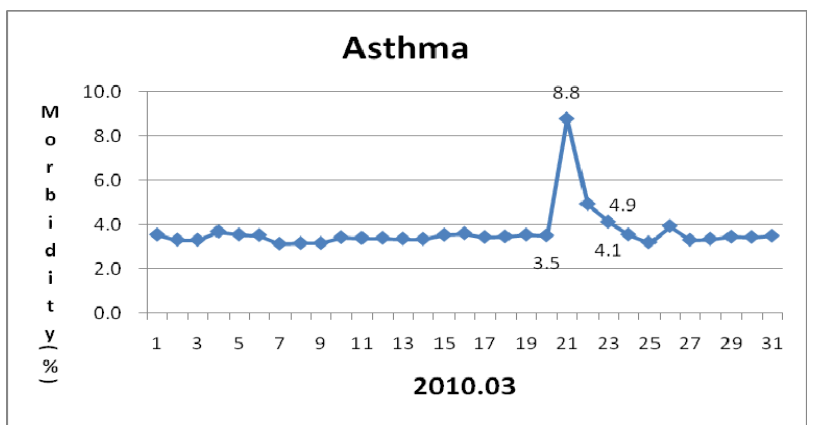

Fig. 14. The morbidities of asthma caused by $\mathrm{PM}_{10}$ during the sandstorm (2010.03.21 23). 


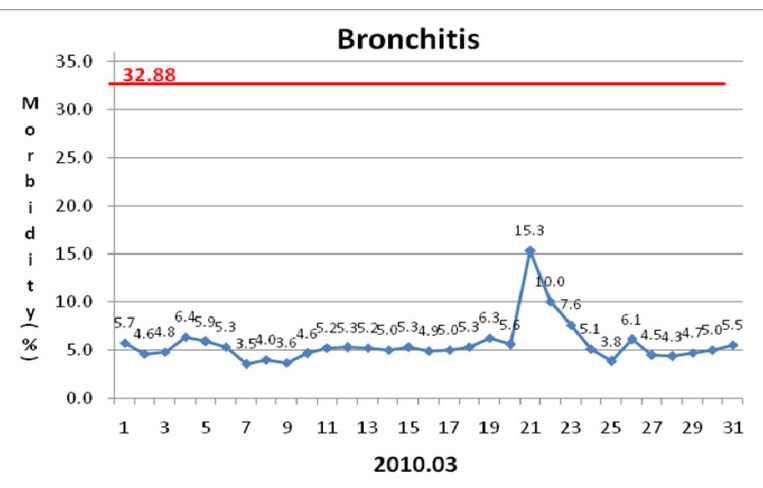

Fig. 15. The overall morbidities of bronchitis during the sandstorm (2010.03.21 23).

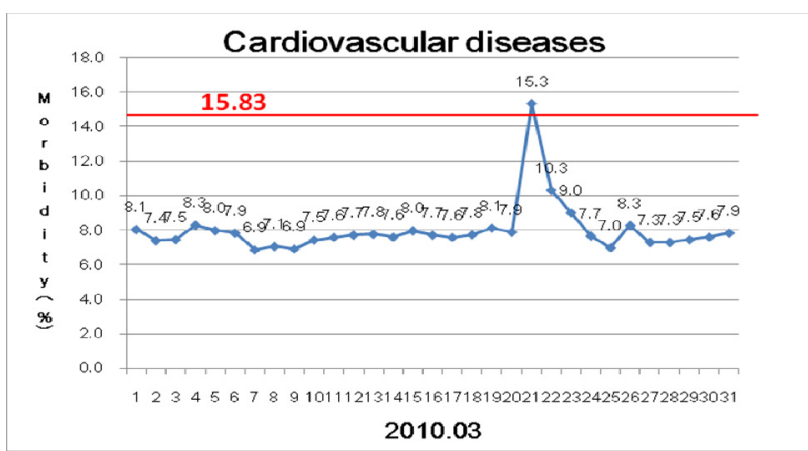

Fig. 16. The overall morbidities of cardiovascular diseases during the sandstorm (2010.03.21 23).

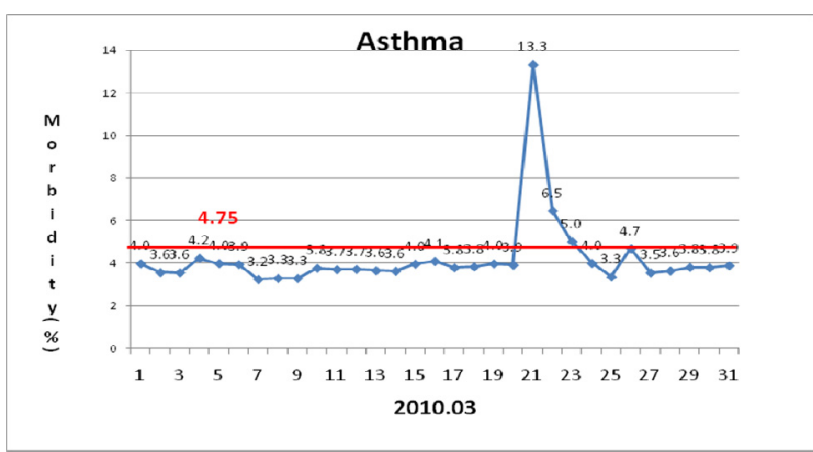

Fig. 17. The overall morbidities of asthma during the sandstorm (2010.03.21 23).

\section{Discussion}

The first case study reveals that the adverse health effects are more severe during winter and spring time. The sensitive population is vulnerable in the period, especially in the south part of Taiwan. The second case study shows the morbidities of cardiovascular diseases and asthma dramatically increase during the sandstorm, primarily due to heavy $\mathrm{TM}_{10}$ in the air. The sensitive population is suggested to stay indoors or wear masks.

\section{ACKNOWLEDGMENT}

The authors would like to thank the National Science Council of the Republic of China (Taiwan) for financially supporting this research under Contract NSC 99-2221 -E-131-010-MY2.

\section{REFERENCES}

[1] CEPA. California Environmental Protection Agency, Office of Environmental Health Hazard Assessment. (2012). A Guide to Health Risk Assessment. [Online]. Available: http://oehha.ca.gov/pdf/HRSguide2001.pdf

[2] K. E. Nachman, M. A. Fox, M. C. Sheehan, T. A. Burke, J. V. Rodricks, and T. J. Woodruff, "Leveraging Epidemiology to Improve Risk Assessment," The Open Epidemiology Journal, vol. 4, pp. 3-29, 2011.

[3] K. C. Raffaele, S. V. Vulimiri, and T. F. Bateson, "Benefits and Barriers to Using Epidemiology Data in Environmental Risk," The Open Epidemiology Journal, vol. 4, pp. 99-105, 2011.

[4] K. F. R. Liu, C. F. Lu, C. W. Chen, and Y. S. Shen, "Applying Bayesian belief networks to health risk assessment," Stoch Environ Res Risk Assess, vol. 26, pp. 451-465, 2012.

[5] K. F. R. Liu, C. F. Lu, C. W. Chen, and Y. S. Shen, "Using Bayesian belief networks to support health risk assessment for sewer workers," International Journal of Environmental Science and Technology, vol.10, pp.385-394, 2013.

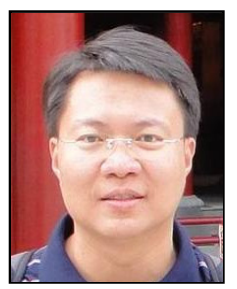

Kevin Liu received the B.S. degree in 1992 and the $\mathrm{Ph} . \mathrm{D}$. degree in 1998, both in civil engineering from National Central University, Taiwan. Currently, he is an Associate Professor in the Department of Safety, Health and Environmental Engineering, Ming Chi University of Technology, Taiwan. His research interest is the use of decision analysis and artificial intelligence techniques to environmental management issues. 